Keywords: Psychosocial treatment, Schizophrenia, Integrated treatment.

\title{
A brief community linkage intervention for veterans with a persistent mental illness and a co-occurring substance abuse disorder
}

\author{
David A. Smelson, Psy.D.*,**,*** \\ Miklos F. Losonczy, M.D., Ph.D.*,**,*** \\ Douglas Ziedonis, M.D., M.P.H. ${ }^{* *}$ \\ Bradley D. Sussner, Ph.D.** \\ Kathy Castles-Fonseca, Psy.D.* \\ Stephanie Rodrigues, B.S.* \\ Anna Kline, Ph.D. ** \\ * Department of Veterans Affairs, New Jersey \\ Health Care System \\ ** Department of Psychiatry, University of \\ Medicine and Dentistry-Robert Wood \\ Johnson Medical School \\ *** Department of Psychiatry, University of \\ Medicine Dentistry-New Jersey Medical \\ School-Newark
}

USA

\begin{abstract}
Objective: Individuals with co-occurring psychiatric and substance abuse problems often exhibit poor outpatient treatment engagement and re-hospitalization following discharge from acute psychiatric services. Although case management can improve treatment engagement and reduce attrition, these services are often delivered indefinitely, limiting the availability of treatment slots. In an effort to reduce re-hospitalization rates and improve outcomes during the transition from inpatient to outpatient treatment, we developed and evaluated Time-Limited Case Management (TLC), an eight-week integrated mental health and substance abuse augmentation intervention.

Method: Sixty-five dually diagnosed veterans admitted to inpatient psychiatric treatment were included in the program evaluation, 32 who received the TLC service in addition to Treatment as Usual (TAU) that began during inpatient treatment and continued after the transition to outpatient services, and a comparison group of 33 who received only TAU without transitional support provided through the TLC augmentation service.

Results: The TLC group had fewer days and episodes of hospitalization at two and six month post-study entry. Furthermore, the TLC group exhibited greater improvements on the Global Assessment of Functioning from baseline to the six-month follow-up.

Conclusion: TLC appears to be an effective transitional augmentation service with benefits that persist beyond the eight weeks of the program. Future research should
\end{abstract}


include a larger and more rigorously controlled trial to confirm the efficacy and unique contributions of the intervention

Received 24 June 2006

Accepted 16 January 2007

\section{Background and Objectives}

Individuals with co-occurring mental illness and substance abuse problem are often non-adherent with treatment and have difficulty engaging in outpatient care. Studies have reported attrition rates of up to $36 \%$ during the transition from inpatient to outpatient treatment ${ }^{1}$. Dropout rates following the initiation of treatment are as alarmingly high and often above $70 \%$, which compromised the success of the sophisticated integrated therapies that have been developed to meet the unique needs of the individuals with a cooccurring disorder ${ }^{2,3}$. Case management augmentation interventions have been used to assist with engaging this population in integrated co-occurring disorders treatment programs, but tend to be based on long-term case management models that often leave mental health systems with far fewer available treatment slots than needed ${ }^{4,5}$.

In response to the large demand for case management services, the field has recently begun to examine time-limited interventions that specifically target vulnerable periods during recovery from psychiatric and substance abuse problems. Several of these new approaches have the potential to reduce the cost and use of acute services, to improve psychosocial and substance abuse outcomes, and to increase linkages with primary care providers ${ }^{6,7}$. It appears that the critical element to successful outcomes for time-limited case management interventions is the con- nection to community-based treatment services, rather than indefinite case management services ${ }^{8}$.

Recognizing that the transition from inpatient psychiatric care to outpatient treatment is often a vulnerable period for people with co-occurring disorders, we developed and evaluated "Time-Limited Case Management" (TLC), an eight-week linkage intervention that combines short-term case management, integrated substance abuse and mental health treatment, and peer support. The TLC approach is consistent with the recommendations included in the Substance Abuse and Mental Health Services Administration (SAMHSA) 2002 report to Congress ${ }^{9}$ and the President's New Freedom Commission on Mental Health report ${ }^{10}$, both of which are critical reports in the United States aimed at improving the fragmented health care system; these reports highlight the need for integrated and coordinated dual diagnosis services. This paper describes the eight-week approach and reports on a preliminary evaluation of its effectiveness.

\section{Methods}

\section{Subjects}

Sixty-five individuals with co-occurring mental illness and substance abuse disorders treated on the Acute Psychiatry unit of the Department of Veterans Affairs New Jer- 
sey Health Care System were included in the project. Thirty-two individuals received the TLC augmentation intervention in addition to treatment as usual (TAU) that consisted of treatment on the Acute Psychiatry unit and a referral for intensive outpatient mental health and substance abuse treatment. A comparison group of thirty-three individuals received only TAU. All of the individuals included in this trial were diagnosed by a doctoral level clinical psychologist (KCF). Subjects for both groups were included if they ${ }^{1}$ : met DSM-IV diagnostic criteria for schizophrenia, schizoaffective disorder, bipolar I disorder or major depressive disorder, and co-occurring substance dependence $^{2}$; had a permanent or transitional residence ${ }^{11}$; were willing and able to use public transportation. Subjects were excluded from the study if they met any of the following criteria: ${ }^{1}$ did not have a chronic mental illness as described above, including substance-induced psychiatric disorder ${ }^{2}$; were homeless; or ${ }^{11}$ had significant medical problems that prevented ambulation.

Because the primary objective of the project was to develop a new treatment intervention, resources were only available to conduct a modest program evaluation rather than a rigorous, randomized controlled research study. Therefore, we used a naturalistic design that included a non-randomized convenience sample who did not sign an informed consent. We later received Institutional Review Board approval to extract demographic, service utilization, and clinical outcome data from the entire sample and to publish the findings.

\section{Treatment as Usual (TAU)}

All subjects in both groups received TAU on the inpatient psychiatric unit followed by a referral to outpatient treatment. Both the inpatient and outpatient programs of the VA New Jersey Health Care System offer psychoeducational skills training, medication management and relapse prevention approaches. Treatment services are primarily delivered in a group format, although patients also participate in individual therapy sessions as needed on the inpatient unit and several times a month during outpatient treatment. The average length of stay on the inpatient psychiatric unit in the fiscal year that the TLC program was developed and evaluated was 13 days. Veterans enrolled in outpatient care are generally in attendance for three days per week for approximately one year.

\section{TLC Treatment}

The TLC augmentation intervention consisted of a comprehensive and integrated set of treatment approaches that include a modified version of Dual Recovery Therapy (DRT), Critical Time Intervention case management (CTI), and peer support, which are discussed below, to meet the diverse needs of patients with co-morbid psychiatric and substance abuse problems. The TLC approach was designed to augment standard inpatient and outpatient treatment. In an effort to increase continuity of care across settings, TLC was delivered for 2 weeks during the patient's stay in Acute Psychiatry and for 6 weeks after their transition to outpatient care. A more detailed description of each component of the intervention is included in Figure 1. Because of the structured and concrete nature of the TLC services, Bachelor's and Master's level clinicians deliver the DRT and CTI interventions with trained consumers doing the peer support. A Doctoral level psychologists performed the diagnostic assessments and assist with the care coordination across inpatient and outpatient treatment. 


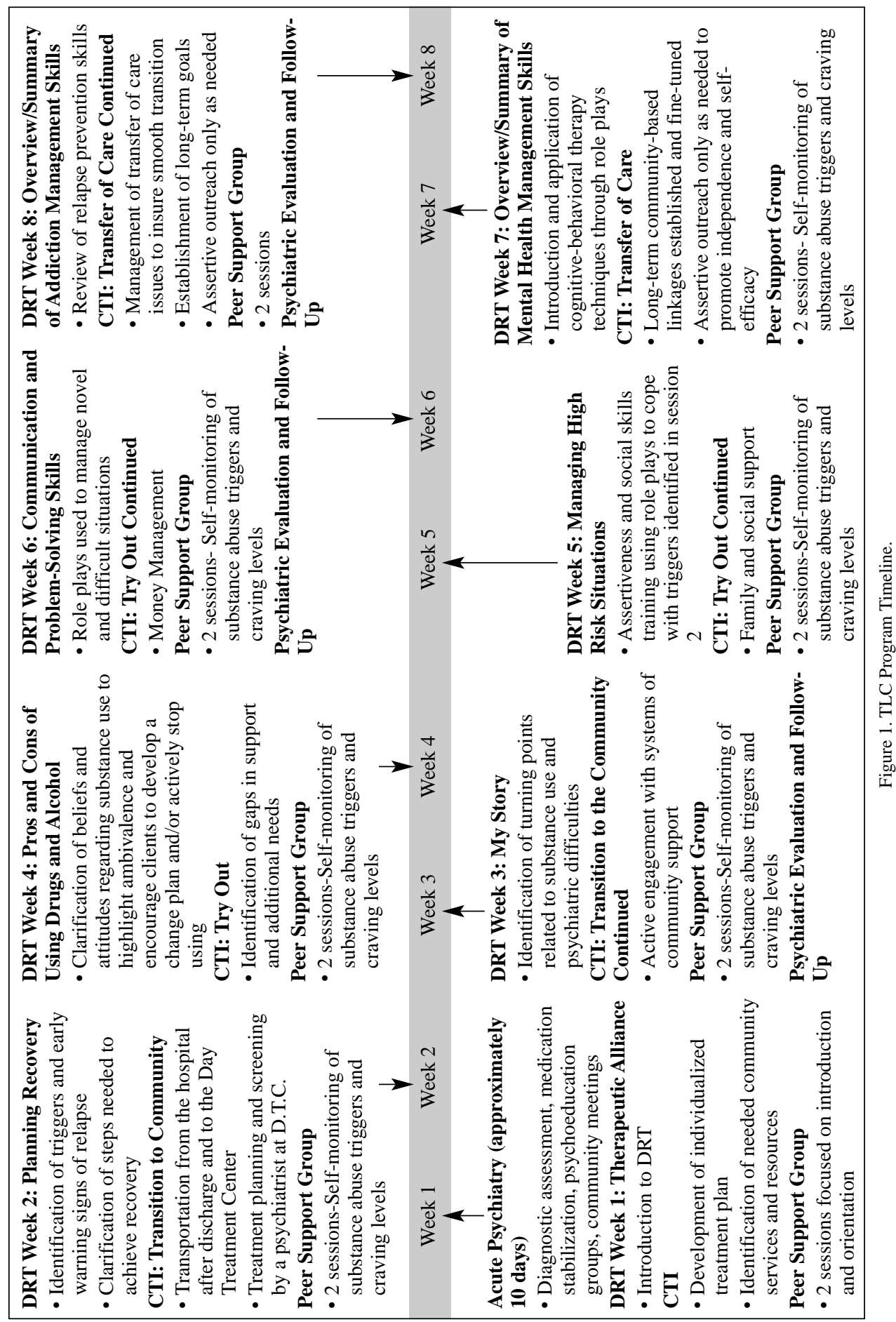




\section{Dual Recovery Therapy}

DRT was originally designed as a twentyweek manual-driven approach for treating individuals with co-occurring psychiatric and substance abuse disorders. The intervention blends relapse prevention, social skills training, motivational enhancement therapy, and the "recovery language" of Twelve-Step Therapy ${ }^{12,13}$. In a number of trials, DRT has contributed to significant improvements in psychiatric and substance abuse outcomes and to better treatment adherence ${ }^{12,14}$. The DRT approach was not modified from the original format for the first six weeks of the TLC intervention. This segment of DRT emphasized motivational enhancement techniques that promote treatment engagement and compliance to minimize the recidivism common among people with co-morbid disorders $^{12,14}$. The remaining two weeks of the eight-week DRT component delivered through TLC is modified from the original format and focused on relapse prevention techniques and psycho-educational skills.

\section{Critical Time Intervention Case}

\section{Management}

The TLC intervention also included one weekly case management session using the Critical Time Intervention approach ${ }^{15}$. This session involved assertive outreach, crisis intervention, transportation assistance, money management, housing assistance and referrals to community programs. On the inpatient psychiatric unit, the two case management sessions (one shortly after arrival on the unit-when ever possible within 72 hours after admission and one prior to discharge) served to assist with the development of a treatment plan and to prepare the individual for the transition to the community.

\section{Peer Support}

TLC also enlisted Peer Support Specialists who were in recovery from co-occurring psychiatric and substance abuse disorders to run two one-hour weekly support group during the six-week outpatient phase. Peer support was included in TLC due to the documented benefits of the intervention in improving social functioning, increasing the use of problem-centered coping strategies, increasing treatment compliance, and reducing rates of re-hospitalization among dually diagnosed patients ${ }^{16,17}$. The peer groups were structured and always began with participants self-monitor craving levels and triggers, except for the first week orientation session. The peers, however, played a key role in the orientation to the treatment programs at each level of care and served as mentors and role models to the individuals entering their recovery journey.

\section{Intervention Schedule}

Those in the TLC group received treatment as usual on the inpatient psychiatric unit along with five extra hours of DRT, CTI and Peer Support programming a week. Upon discharge, these individuals received standard outpatient mental health and substance abuse treatment along with two DRT groups, one individual CTI session, and two peer support session each week for six weeks. The same case managers provided TLC services on both the inpatient and outpatient units to foster continuity across levels of care. Case managers were responsible for providing input into treatment plans and for assisting with the execution of the treatment goals during and after the transition from inpatient treatment. Throughout the eight weeks of the program, the TLC case managers functioned as secondary providers to the inpatient and outpatient staffs to reinforce the idea that the purpose of TLC was to assist with the transition from inpatient to outpatient care, not to replace existing services. By emphasizing a supportive, rather than a leading role in patient care, the TLC 
program enabled the outpatient staff to easily assume the service coordination responsibilities by the end of the eight-week treatment period.

\section{Assessments}

Assessments were conducted at baseline within approximately seventy-two hours after admission to the inpatient psychiatric unit, and at two months and six months after study entry. Subjects completed the Addiction Severity Index ${ }^{18}$ to determine drug use and functioning in various life domains (e.g., criminal justice, family/social, employment) during the 30 days prior to the assessment. Because the study had a broad inclusion criteria, patients were also administered the Behavioral and Symptom Identification Scale (BASIS-32), a 32 item self-report questionnaire that has been validated and found reliable in both inpatient and outpatient settings ${ }^{19}$. The BASIS accommodates a variety of diagnostic categories with subscales that assess "relation to self/others," "Depression/Anxiety," Daily Living/Role Functioning," Impulsive/Addictive Behavior," "Psychosis"19. This instrument measures the change in self-reported symptom and problem difficulty over the course of treatment. The Global Assessment of Functioning $(\mathrm{GAF})^{20}$ was used to measure general psychiatric functioning. The GAF is a widely used instrument that is part of the Diagnostic and Statistical Manual for psychiatric Disorders $4^{\text {th }}$ addition and a VA performance measure. Service utilization data was obtained through chart reviews in the VA's electronic medical record system.

\section{Statistical Analyses}

Student's independent samples t-tests were used to measure group baseline differences on age, pre-treatment hospitalization, length of current hospitalization, addiction severity, and psychiatric functioning. Repeat- ed measures ANOVAs were used to compare the groups on days and episodes of hospitalization in the two and six months before and two and six months after study entry. Due to the large difference in the percentage of subjects in the TLC and comparison groups that were available for the two-month poststudy entry assessment period (25.0\% vs. $81.8 \%$, respectively), between groups analyses comparing scores on the clinical outcome measures at baseline and at the two-month follow-up were not performed. We were, however, more successful in locating subjects for the six-month followup assessments. This allowed us to perform repeated measures ANOVAs to compare the change in the groups over time on the GAF, the BASIS-32 subscales, and the ASI drug use subscales.

\section{Results}

At baseline, there were no significant differences between the TLC and TAU groups in terms of age, substance use in the 30 days prior to the assessment, GAF score and the BASIS-32 subscales (see Table I). Individuals in the treatment group, however, were hospitalized for more days in the two months prior to completing the initial study evaluation.

Post-discharge outcomes indicated that individuals who received the TLC service exhibited greater reductions than the comparison group in the number of hospitalization days in the two months after their current hospitalization compared to the two months before their admission, $F(1,58)=$ $12.8, \mathrm{p}<.01$. Greater reductions in hospitalization days for the TLC group were also observed for the six months before and six months after their current psychiatric admis- 
Table I

Baseline Demographic and Clinical Comparisons.

\begin{tabular}{lcc} 
Means \pm SD at Baseline & $\begin{array}{c}\text { TLC Treatment } \\
(\mathrm{N}=33)\end{array}$ & $\begin{array}{c}\text { Comparison Group } \\
(\mathrm{N}=37)\end{array}$ \\
\hline Age & $45.4 \pm 6.6$ & $45.7 \pm 6.6$ \\
Global Assessment of Functioning & $44.9 \pm 8.4$ & $45.0 \pm 8.2$ \\
Hospital Days Current Admission & $20.0 \pm 18.6$ & $16.0 \pm 10.5$ \\
*Hospital Days 2 Months Pretreatment & $21.9 \pm 18.1$ & $14.1 \pm 11.3$ \\
Hospital Days 6 Months Pretreatment & $40.7 \pm 34.9$ & $30.5 \pm 36.0$ \\
\hline Alcohol Use 30 Days Pretreatment & $5.9 \pm 8.6$ & $4.3 \pm 7.5$ \\
Heroin Use 30 Days Pretreatment & $.7 \pm 2.1$ & $2.1 \pm 6.6$ \\
Cocaine Use 30 Days Pretreatment & $5.6 \pm 7.7$ & $4.3 \pm 7.6$ \\
Cannabis Use 30 Days Pretreatment & $.1 \pm .4$ & $1.2 \pm 4.3$ \\
Days Intoxicated 30 Days Pretreatment & $4.1 \pm 7.1$ & $3.7 \pm 7.0$ \\
BASIS-32 Relation to Self/Others & $1.7 \pm 1.2$ & $2.0 \pm 1.0$ \\
BASIS-32 Depression/Anxiety & $2.0 \pm 1.3$ & $2.4 \pm 1.1$ \\
BASIS-32 Daily Living/Role Functioning & $1.7 \pm .92$ & $2.2 \pm 1.1$ \\
BASIS-32 Impulsive/Addictive Behavior & $1.4 \pm 1.1$ & $1.1 \pm .9$ \\
BASIS-32 Psychosis & $1.6 \pm 1.1$ & $1.4 \pm 1.0$ \\
BASIS-32 Average & $1.8 \pm 1.1$ & $1.9 \pm$ \\
\hline
\end{tabular}

sion, $F(1,58)=4.02, \mathrm{p}=.05$. In addition to the reduction in hospitalization days, members of the TLC group experienced a greater reduction in the number of episodes of hospitalization from the two months before study entry to the two months after study entry, $F(1$, $58)=6.33, \mathrm{p}=.02$, as well as the six months before entering the study to the six months after study entry, $F(1,58)=7.14, \mathrm{p}=.01$.

Results from the GAF indicated that subjects in the TLC group exhibited greater improvement in global psychiatric functioning than the comparison group over the course of the study, $F(1,39)=4.1, \mathrm{p}=.05$. The interactions of group by time for the subscales of the BASIS-32 were not significant: "Relation to Self and Others," $F(1,33)=.9, \mathrm{p}=.35$, "Depression/Anxiety," $F(1,33)=.6, \mathrm{p}=.45$, "Daily Living/Role Functioning," $F(1,33)=$ $.9, \mathrm{p}=.35$, "Impulsive/Addictive Behavior," $F(1,33)=.16, \mathrm{p}=.72$, "Psychosis," $F(1,33)=$ $.16, \mathrm{p}=.70$, and "Average," $F(1,33)=1.3, \mathrm{p}=$ .27. In terms of substance abuse outcomes, the TLC group displayed a trend toward greater reductions in cannabis use from the baseline to the six-month follow up assessment than the comparison group, $F(1,38)=3.4, \mathrm{p}=.07$. Differences in change for alcohol, $F(1,38)=$ $1.27, \mathrm{p}=.27$, cocaine, $F(1,38)=.96, \mathrm{p}=.33$, and heroin use, $F(1,38)=.1, \mathrm{p}=.74$, were not significant.

\section{Conclusions}

The findings of this study support the main objective that individuals who received the eight-week TLC augmentation community linkage intervention would have fewer days and episodes of acute inpatient psychiatric readmission during the eight weeks of the TLC intervention than subjects who were discharged as usual with a referral to outpatient treatment without additional support. With regard to clinical outcomes, subjects in the TLC group exhibited greater improvements on the GAF than the compar- 
ison group. It should be pointed out, however, that while some researchers have expressed concerns over the GAF's reliability as an outcome measure because it is a unidimensional rating scale for assessing functional outcome ${ }^{21}$, other research involving case management interventions suggest that the GAF may be more sensitive to treatment effects then measures involving symptom severity since the latter is often an unstable trait that is heavily influences by a variety of day-to-day circumstances ${ }^{5}$. Those in the TLC group also exhibited less cannabis use than the comparison group at a level that approached statistical significance.

The most encouraging finding of this study was that the significantly greater reduction in hospitalization days and episodes was still evident six months after study entry. This finding is consistent with the work of Drake et $a .^{22}$, and others using long-term models, but is in stark contrast to the findings of Havassy, et al. ${ }^{23}$ who observed that intensive and expanded brokerage case management were largely ineffective for decreasing hospitalizations among dually diagnosed subjects. It is possible that TLC was successful in reducing re-hospitalization because the program's staff directly addressed the challenges associated with co-morbid disorders through DRT in addition to offering case management services.

Despite the fact that the TLC service resulted in robust reductions in the use of acute inpatient services, it had less of an impact on psychiatric and substance abuse outcomes. Although we found large improvements in global psychiatric functioning and some reduction in cannabis use for the TLC group, we observed little improvement on the subscales of the BASIS32 or reductions in alcohol and cocaine use. This is consistent with the conclusions of Holloway et $a l .{ }^{24}$, who reported that of the case management studies they reviewed a majority failed to find statistically significant improvements in symptomatology. Though it is possible that our relatively small sample size limited the statistical power of the study, it is also plausible that six months is an insufficient period of time in which to expect psychiatric and substance abuse problems to stabilize in dually diagnosed subjects.

Although traditional case management has helped individuals in the transition from inpatient hospitalization to outpatient care, TLC appears to offer several advantages for this population. Unlike many traditional case management programs that are offered indefinitely, TLC is delivered for only eight weeks. This limited time frame allows clinicians to meet the needs of a greater number of individuals (approximately 8-10 new clients every eight weeks). Granholm et al. ${ }^{11}$ recently observed that a brief (up to 24 weeks) intervention for dually-diagnosed individuals can result in long-term reductions in hospital use. Our preliminary findings suggest that extended treatment benefits can be obtained from an intervention one-third as long. The TLC approach also emphasizes early and consistent relationships with clinicians and peers that begin during psychiatric hospitalization to foster a smooth transition across different levels of care. The on-going presence of TLC clinicians during and after psychiatric hospitalization can add a sense of familiarity to outpatient care and minimize the anxiety often associated with beginning a new form of treatment in a new setting. Another important element of the TLC model is that it simultaneously addresses the challenges associated with psychiatric and substance abuse disorders based on the premise that each set of problems has an impact on the others $^{25-28}$. Too often these problems are 
conceptualized and treated by separate teams of clinicians resulting in service fragmentation $^{29}$.

Although we believe that the positive results we observed were attributable to the unique characteristics of the TLC augmentation service, a number of methodological limitations were associated with the naturalistic nature of the study. Because randomization was not used to assign subjects into treatment groups, it is possible that preexisting differences between the groups on such issues as insight into the presence of psychiatric and substance abuse disorders, recognition of the need for treatment, and motivation to address personal problems may have contributed to the results that were obtained. Furthermore, although a doctorallevel psychologist assessed all of the individuals entering the TLC service, a structured diagnostic instrument would have been beneficial to substantiate the diagnosis and inclusion into the study. In order to address these limitations, future research on TLC should include a large randomized controlled design matched for the additional attention received by the TLC augmentation services. Finally, the TLC intervention needs to be implemented and studied outside of the VA system to determine its efficacy in other more diverse systems of healthcare.

\section{Acknowledgements:}

This work was supported by grants from Substance Abuse and Mental Health Service Administration (H79 TI16576), and a VA HSR\&D Merit Review (MR-2-09499) to DS, and National Institute of Drug Abuse (R01 DA15978 and RO1 DA 15537) to DZ. We also wish to thank the VA New Jersey Health Care System and the VISN 3 Mental
Illness Research Education and Clinical Center. Parts of this article were presented at the New Clinical Drug Evaluation Unit Meeting, Boca Raton, Florida, June 2004.

\section{References}

1. Nuttbrock LA, Rahav M, Rivera JJ, et al. Outcomes of homeless mentally ill chemical abusers in community residences and a therapeutic community. Psychiatr Serv 1997; 49: 68-76.

2. Carey KB: Substance use reduction in the context of outpatient treatment: A collaborative, motivational, harm reduction approach. Community Ment Hlt J 1996; 32: 291306.

3. Rahav M, Rivera JJ, Nuttbrock L. Characteristic and treatment of homeless, mentally ill, chemical-abusing men. J Psychoactive Drugs 1995; 27: 91-93.

4. Drake RE, Yovetich NA, Bebout RR, et al. Integrated treatment for dually diagnosed homeless adult. J Nerv Ment Dis 1997; 185: 298-305.

5. Mueser KT, Bond GR, Drake RE, et al. Models of community care for severe mental illness: A review of research on case management. Schizophrenia Bull 1998; 24: 37-74.

6. Witbeck G, Hornfield S, Dalack GW. Emergency room outreach to chronically addicted individuals: a pilot study. Journal of Subst Abuse Treat 2000; 19: 39-43.

7. Okin RL, Boccellari A, Azocar F, et al. The effects of clinical case management on hospital service use among ER frequent users. Am J of Emerg Med 2000; 18: 603-608.

8. Rosenheck RA, Dennis D. Time-limited assertive community treatment for homeless persons with severe mental illness. Arch Gen Psychiat 2001; 58: 1073-1080.

9. Substance Abuse and Mental Health Services Administration. Report to Congress on the Prevention and Treatment of Co-Occuring Substance Abuse Disorders and Mental Disorders. Washington, D.C. 2002.

10. President's New Freedom Commission on Mental Health. Achieving the promise: Transforming mental health care in America. Washington, D.C. 2003.

11. Granholm E, Anthenelli R, Monteiro R, et al. Brief integrated outpatient dual-diagnosis treatment reduced psychiatric hospitalizations. Am J Addict 2003; 12: 306-313. 
12. Ziedonis DM \& Fisher W. Motivation-based assessment and treatment of substance abuse in patients with schizophrenia. Directions Psychiat 1996; 16: 1-8.

13. Ziedonis D, Stern R. Dual Recovery Therapy for Schizophrenia and Substance Abuse. PsychiatAnn 2001; 31: 255-264.

14. Ziedonis DM \& Trudeau K. Motivation to quit using substances among individuals with schizophrenia: Implications for a motivation based treatment model. Schizophrenia Bull 1997; 23: 229-238.

15. Susser E, Betne P, Valencia E, et al. Injection drug use among homeless adults with severe mental illness. Am J Public Health 1997; 5: 854-856.

16. Davidson L, Chinman M, Kloos B, et al. Peer support among individuals with severe mental illness: A review of the evidence. Clin Psychol-Sci Pr 1999; 6: 165187.

17. Wilson ME, Flanagan S, Rynders C. The Friends program: A peer support group model for individuals with a psychiatric disability. Psychiat Rehabil J 1999; 22: 239247.

18. McLellan AT, Luborsky L, Woody G. An improved diagnostic evaluation instrument for substance abuse patients: the addiction severity index. J Nerv Ment Dis 1980; 168: 26-33.

19. Eisen SV, Wilcox M, Leff HS, et al. Assessing behavioral health outcomes in outpatient programs: reliability and validity of the BASIS-32. J Behav Health Serv Res 1999; 26: 5-17.

20. Diagnostic and Statistical Manual of Mental Disorders - Fourth Edition (DSM-IV) (1994), American Psychiatric Association, Washington D.C.

21. Moos R, McCoy L, Moos B. Global assessment of functioning scores: Clinicians ratings do not predict substance abuse patients one year outcomes. QUERI Quarterly 1999; 1(3): 2-3.
22. Drake RE, McHugo GJ, Clark RE, et al. Assertive community treatment for patients with co-occurring severe mental illness and substance use disorder: a clinical trial. Am J Orthopsychiat 1998; 68: 201-215.

23. Havassy BE, Shopshire MS, \& Quigley LA. Effects of substance dependence on outcomes of patients in a randomized trial of two case management models. Psychiat Serv 2000; 51: 639-644.

24. Holloway F, Oliver N, Collins, E, et al. Case management: a critical review of the outcome literature. Eur Psychiat 1995; 10: 113-128.

25. Dixon L. Dual diagnosis of substance abuse in schizophrenia: prevalence and impact on outcomes. Schizophr Res 1999; 35: 93-100 Suppl. S Mar 1.

26. Kosten TR, Ziedonis DM. Substance abuse and schizophrenia: editors' introduction. Schizophrenia Bull 1997; 23: 181-186.

27. Reis R, Mullen M, Cox G. Symptom severity and utilization of treatment resources among dually diagnosed inpatients. Hosp Community Psychiat 1994; 45: 562-568.

28. Salloum IM, Thase ME. Impact of substance abuse on the course and treatment of bipolar disorder. Bipolar Disord 2000; 2: 269-280.

29. Rosenheck RA, Resnick SG, Morrissey JP. Closing service system gaps for homeless clients with a dual diagnosis: integrated team and interagency cooperation. J Ment Health Policy 2003; 6: 77-87.

Address for correspondence:

David A. Smelson, Psy.D.

Department of Veterans Affairs

New Jersey Health Care System, Lyons Campus

Mental Health and Behavioral Sciences (Bldg. 143)

151 Knollcroft Road

Lyons, N.J. 07939-5000

Telephone: (908) 6470180 Ext. 5122

Email: david.smelson@med.va.gov 